\title{
Reconnection and energetic particles at the edge of the exterior cusp
}

\author{
T. Asikainen and K. Mursula
}

Department of Physical Sciences, PoBox 3000, FIN-90014 University of Oulu, Finland

Received: 20 January 2006 - Revised: 10 May 2006 - Accepted: 24 May 2006 - Published: 9 August 2006

\begin{abstract}
In this paper we study flux transfer events (FTE) observed at the post-noon edge of the exterior cusp region by Cluster satellites. During the outbound dayside orbit on 2 February 2003, intense bursts of energetic particles were observed in close conjuction with magnetic field FTE signatures by the RAPID instrument onboard the Cluster 4 . The pitch-angle distribution of the particles showed that the enhancements consist of particles flowing antiparallel to the magnetosheath field lines away from the expected reconnection site to the exterior cusp. At the same time Cluster 3 observed enhancements of energetic particles deeper in the exterior cusp with a delay of about $40 \mathrm{~s}$ to the Cluster 4 enhancements. The estimated maximum energy gain per particle by reconnection remains below $1 \mathrm{keV}$, thus clearly below the tens to hundreds of $\mathrm{keV}$ energy range observed by the RAPID instrument. These observations support the earlier statistical result of the magnetospheric origin of energetic particles in the exterior cusp. Reconnection near the exterior cusp partly releases the particles in the closed field lines of the adjacent HLPS region into the exterior cusp.
\end{abstract}

Keywords. Magnetospheric physics (Energetic particles, trapped; Magnetopause, cusp and boundary layers) - Space plasma physics (Magnetic reconnection)

\section{Introduction}

Recent satellite missions, in particular Polar and Cluster, have demonstrated the existence of energetic particles in the exterior cusp region (Chen et al., 1998; Asikainen and Mursula, 2005). While previously it was thought that the highlatitude regions near the topological cusp in the dayside magnetosphere could not stably trap particles (Roederer, 1970) it was later discovered that the magnetic field geometry around the cusp can trap energetic particles (Sheldon et al., 1998). The energetic particles in the exterior cusp have been studied by a number of authors. Chen et al. (1998) and Fritz (2001) showed that energetic particles indeed appear in the diamag-

Correspondence to: T. Asikainen

(timo.asikainen@oulu.fi) netic cavity of the exterior cusp where the magnetic field is extremely variable and the field strength is greatly depleted due to the high density plasma. Chen et al. (1998) found that the power of magnetic fluctuations correlated with the flux of energetic particles, and proposed that the magnetic fluctuations accelerate a portion of thermal magnetosheath plasma to high energies in the cusp diamagnetic cavity.

We have recently presented a different mechanism to explain the existence of energetic particles in the exterior cusp (Asikainen and Mursula, 2005). We performed a statistical analysis of energetic particle fluxes in the exterior cusp and the adjacent high-latitude dayside plasmasheet (HLPS) observed by Cluster satellites. Comparing particle fluxes in the two regions with geomagnetic activity and solar wind/IMF parameters we concluded that the majority of energetic particles in both of these two regions originate in the Earth's magnetotail where they are accelerated by substorm processes. Energetic particles, especially electrons above $20 \mathrm{keV}$ (all RAPID electrons) and protons above some $100 \mathrm{keV}$ are transported from the HLPS to the exterior cusp mainly by direct diffusion. We also found evidence suggesting that reconnection is a key factor in transporting protons below $100 \mathrm{keV}$ to the exterior cusp by controlling their release from the closed field lines.

In this paper we present a detailed case study of an exterior cusp pass by Cluster satellites. We will show that reconnection can release significant amounts of trapped energetic particles from the HLPS to the exterior cusp. The paper is organised as follows. In Sect. 2 we briefly review the instrumentation used. Section 3 contains an overview of the studied cusp pass. Section 4 presents detailed observations of reconnection at the exterior cusp and simultaneous enhancements of energetic particle fluxes. Observations are discussed in Sect. 5 and conclusions given in Sect. 6.

\section{Instrumentation}

In this work we use energetic particle data from the RAPID (Research with Adaptive Particle Imaging Detectors) instruments on board the Cluster satellites (Wilken et al., 1997). The IES sensor of RAPID measures electrons in

Published by Copernicus GmbH on behalf of the European Geosciences Union. 

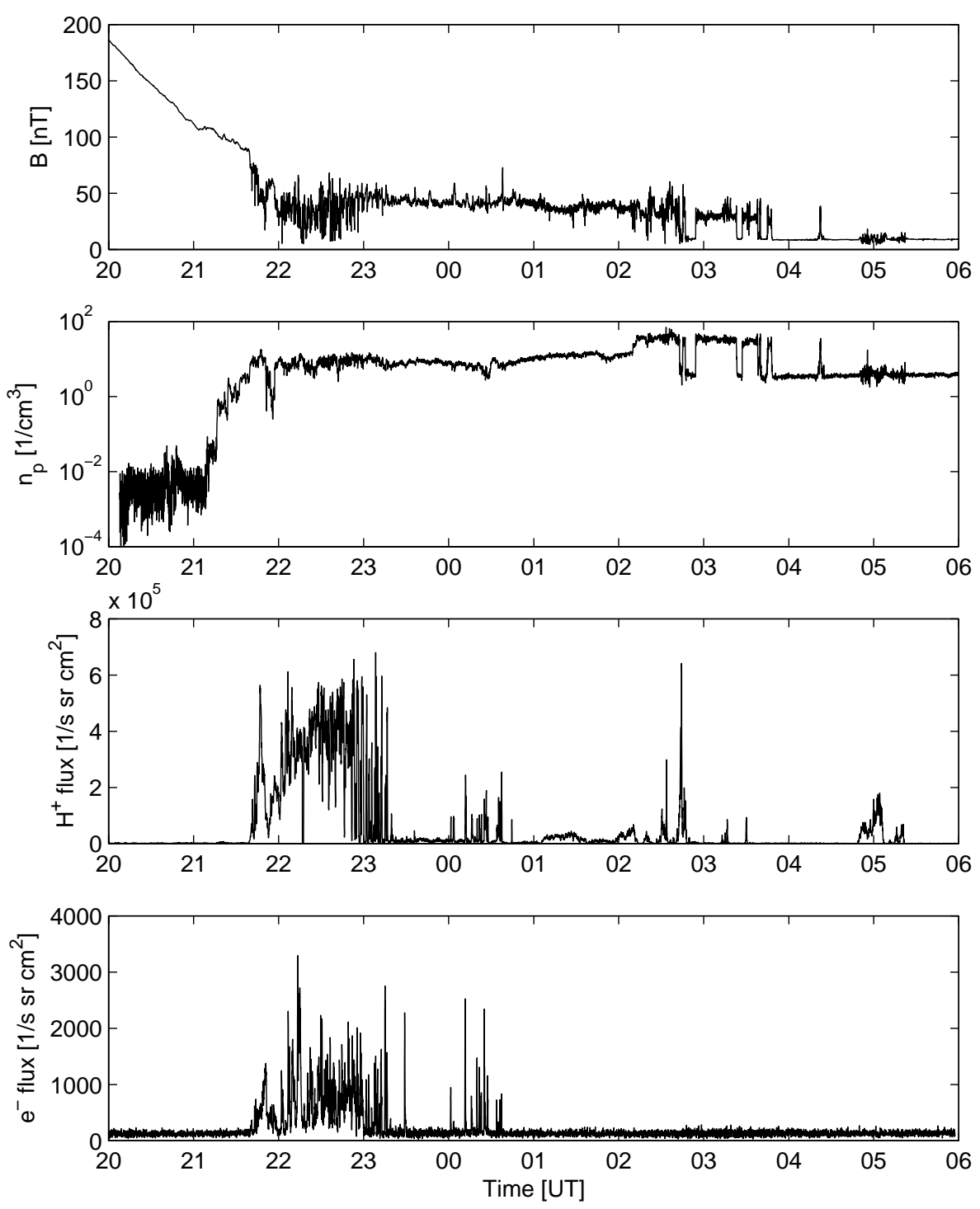

Fig. 1. Overview of Cluster 4 data on 2-3 February 2003. Panels from top to bottom depict the magnetic field intensity, plasma proton density, and the total fluxes of energetic protons and electrons measured by the RAPID instrument.

the energy range $20-400 \mathrm{keV}$ and the IIMS sensor protons, helium ions and heavier ions in the CNO (carbon, nitrogen, oxygen) group. The energy range for protons and helium ions is $40 \mathrm{keV}-4000 \mathrm{keV}$ and for the heavier ions from $10 \mathrm{keV} /$ nucleon to $1500 \mathrm{keV}$. Both sensors can measure the three-dimensional angular distribution of particles during the 4-s satellite rotation period. The azimuthal (spin) plane is divided into 16 sectors yielding an azimuthal resolution of $22.5^{\circ}$. The polar resolution is $20^{\circ}$ for electrons and $15^{\circ}$ for ions. Moreover, we use magnetic field data of the FGM (Flux Gate Magnetometer) instrument (Balogh et al., 1997) and plasma density data of CIS instrument (Rème et al., 1997) at 4-s resolution.

\section{Overview}

In this paper we study the pass of Cluster satellites through the exterior cusp on 2-3 February 2003. During this time the configuration of the four satellites was such that $\mathrm{C} 4$ was leading (largest $\mathrm{X}$ ) and $\mathrm{C} 3$ trailing (smallest $\mathrm{X}$ ), lagging about 30 min. $\mathrm{C} 1$ and $\mathrm{C} 2$ were quite close to $\mathrm{C} 4$. Figure 1 shows an overview of Cluster 4 magnetic field, plasma density and energetic protons and energetic electrons from 20:00 UT to 06:00 UT. At the beginning of the time interval $\mathrm{C} 4$ was travelling from the tail lobe/HLBL region towards the dayside inside the magnetopause, as verified by the stable magnetic field and low plasma density. At about 21:45 UT the magnetic field strength dropped rapidly and the field became highly variable. By that time the plasma density had 

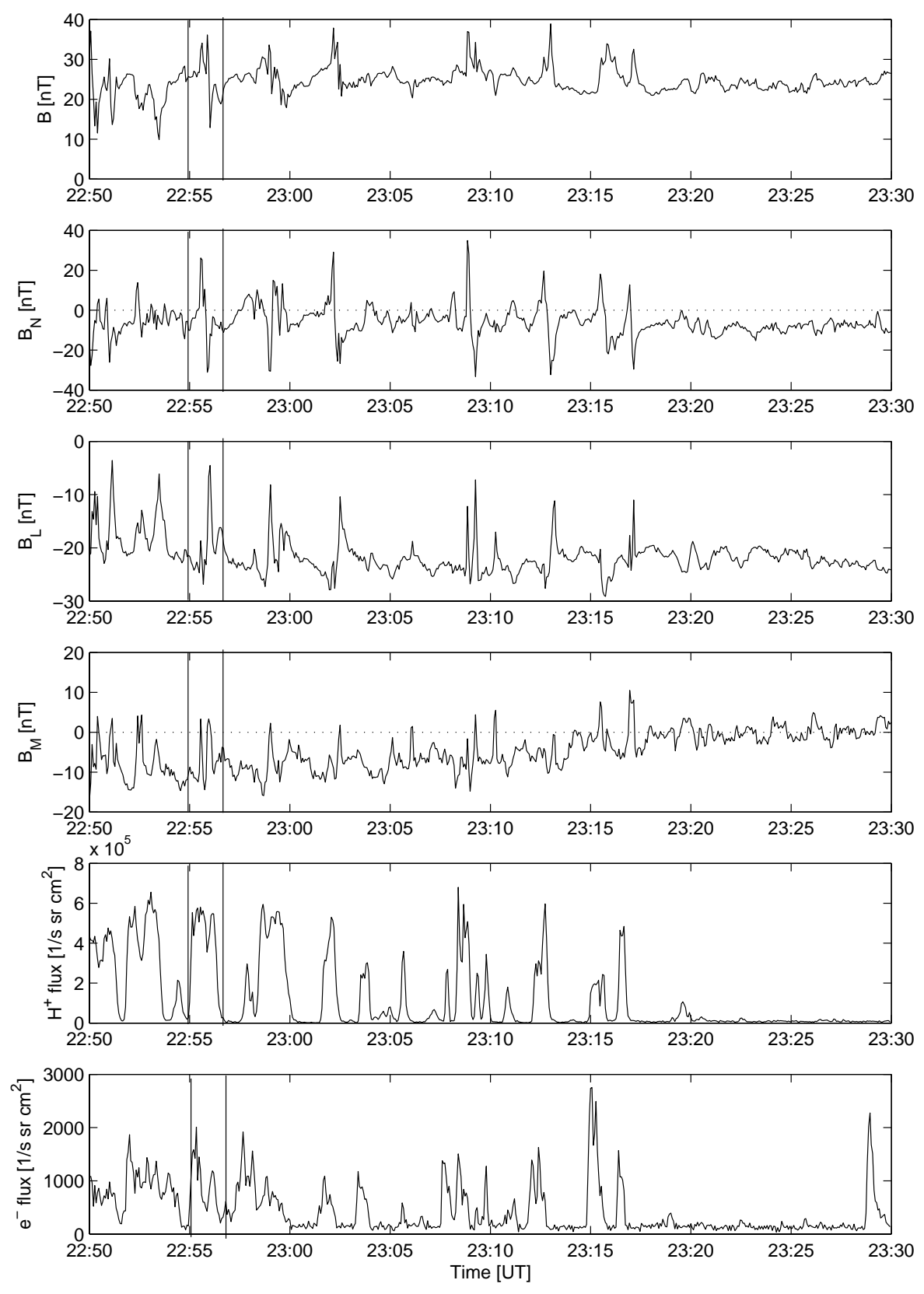

Fig. 2. Expanded view of Cluster 4 data in 22:50-23:30 UT. Panels from top to bottom depict the magnetic field intensity, the $B_{N}$, $B_{L}$, $B_{M}$ components (in boundary normal coordinate system where $\boldsymbol{n}=(0.352,0.379,0.856)$ in GSE system), and the total fluxes of energetic protons and electrons. The two vertical lines denote the time interval of the angular distribution in Fig. 4.

increased close to the magnetosheath level. Based on these signatures we can say that the satellite entered the exterior cusp at 21:45 UT. After about 23:20 UT the magnetic field fluctuations decreased and the field strength became more stable, indicating that $\mathrm{C} 4$ had finally exited the exterior cusp and entered the magnetosheath by this time. Note that during its stay within the exterior cusp, C4 detected the largest flux of energetic particles (both ions and electrons). (C3, not shown, visited the exterior cusp from about 22:10 UT to 23:30 UT). Later, C4, after numerous crossings through the bow shock since 02:45 UT, finally entered the solar wind at about 03:45 UT .

\section{Detailed observations}

The most interesting period in $\mathrm{C} 4$ data for this study is the time interval from about 22:50 UT to 23:30 UT, i.e., the 

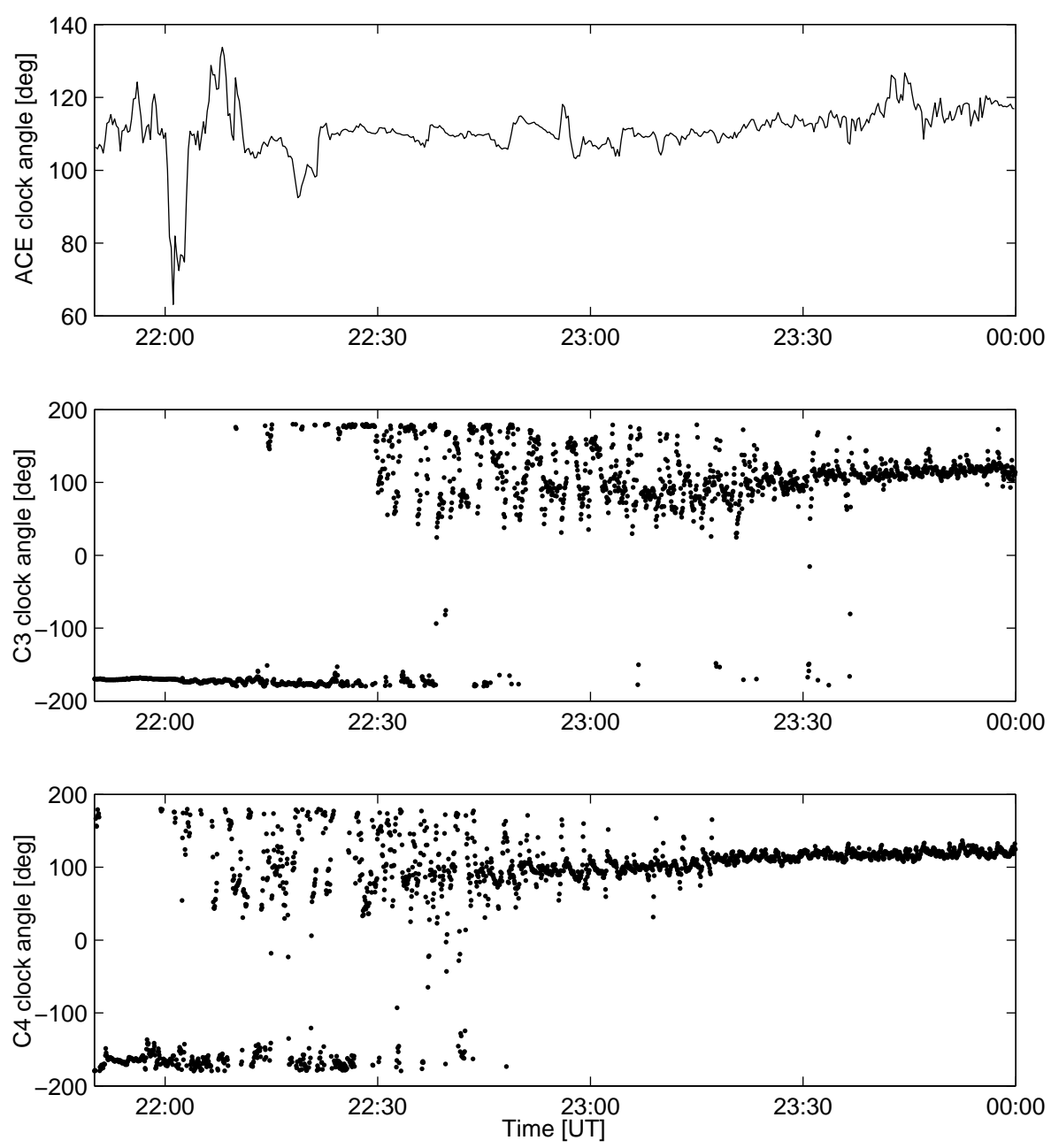

Fig. 3. Clock angles of ACE (delayed by $50 \mathrm{~min}$ ), C3 and C4 satellites.

latter part of $\mathrm{C} 4$ cusp visit and the transition from cusp to magnetosheath (magnetopause crossing). Figure 2 shows an expanded view of $\mathrm{C} 4$ magnetic field and energetic particle data for this interval. The magnetic field is expressed in the boundary normal coordinate system. The unit vectors are defined so that vector $\boldsymbol{m}=\boldsymbol{n} \times \boldsymbol{z}_{G S M}$ and $\boldsymbol{l}=\boldsymbol{m} \times \boldsymbol{n}$. The normal vector $\boldsymbol{n}=(0.352,0.379,0.856)$ (GSE) was estimated by assuming a planar boundary and using boundary crossing times and corresponding positions of the four satellites. The individual boundary crossing times were identified from the energetic ion measurements of the four satellites (see also Mursula et al., 2005). Minimum variance analysis of the magnetic field did not yield a good result for the boundary normal in this case due to the very noisy field on both sides of the boundary. In Fig. 2 one can clearly see a number of bipolar signatures in the normal component of magnetic field from 22:55 UT to 23:17 UT. These signatures seem to occur quasiperiodically with a period of about 3-4 min. It has been known for quite some time that the flux transfer events (FTE) associated with temporally variable reconnection at the dayside magnetopause give rise to such bipolar signatures in the magnetic field, especially in the component along the local magnetopause normal (Russell and Elphic, 1978).

The IMF observed by ACE spacecraft (located at L1 point with an average delay time of about $50 \mathrm{~min}$ ) during the interval from 22:50 UT to 23:30 UT was rather steady with an average of $(6,10,-3) \mathrm{nT}$ in GSM coordinates, yielding an average clock angle (the angle between the projection of IMF vector to GSM YZ plane and GSM Z axis) of about $107^{\circ}$ (see Fig. 3). At the same time interval the local magnetic field observed by $\mathrm{C} 4$ was on average about $(16,39,-9) \mathrm{nT}$ in GSM coordinates, which is rather close to the direction of the IMF field lines, taking into account that they are shocked in the bow shock and draped over the magnetopause. This verifies that $\mathrm{C} 4$ was outside the magnetopause on shocked IMF field lines at this time. Had it been inside the magnetosphere on closed field lines, the $\mathrm{Y}$ (and $\mathrm{X}$ ) component should be negative. The local magnetic field clock angle of 


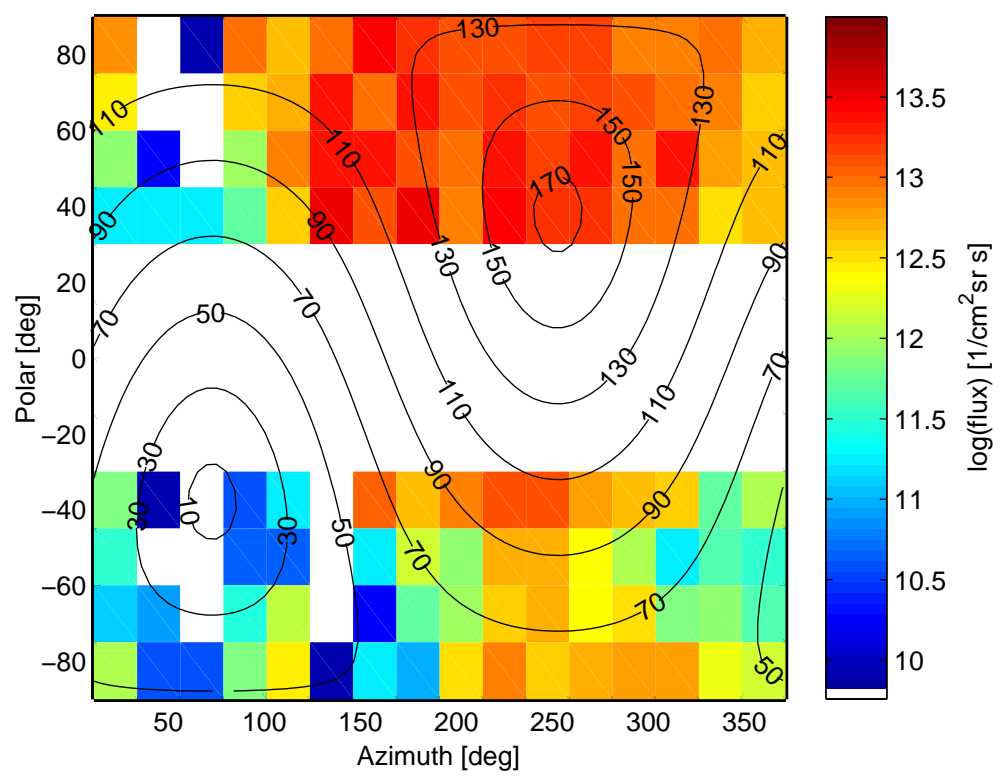

Fig. 4. Angular distribution of protons in GSE coordinates in 22:55:21-22:57:29 UT (the burst noted in Fig. 2). The angles represent the flow direction of the particles. The maximum fluxes are observed coming from duskward of the Sun-Earth line, while very low fluxes are observed coming from the opposite direction. The empty area between polar angles from $-30^{\circ}$ to $30^{\circ}$ is due to the corrupted central sensor heads of the RAPID IIMS instrument. Pitch angle contours corresponding to average magnetic field $\boldsymbol{B}_{G S E}=(9.7,31,-25) \mathrm{nT}$ observed by C4 are also shown.

C3 satellite shows variations between IMF directed field and nearly southward directed field $\left( \pm 180^{\circ}\right.$ clock angle) which is more typical to the exterior cusp. This implies that during the time interval $\mathrm{C} 3$ was very close to the boundary between exterior cusp and magnetosheath and even crossed the boundary a number of times. During this IMF orientation the antiparallel reconnection occurs somewhat equatorward and duskward of the cusp funnel, close to the so called top of the field line position. The position of $\mathrm{C} 4$ was about (4.5, $2,9.3) R_{E}$ (in GSM), i.e., close to the position of expected antiparallel reconnection site. On the basis of the bipolar signatures in the magnetic field and the position of the satellite relative to the expected reconnection site it is clear that the signatures are FTEs caused by transient reconnection at the dayside. The (+/-) polarity of the $B_{N}$ signatures indicates that the FTE pulses are moving away from the reconnection site (which is equatorward of the satellite position) towards the exterior cusp.

Associated with the FTE signatures the RAPID instrument detects strong bursts of energetic ions and electrons (see Fig. 2). (A similar relation between the FTE signatures and energetic particles was presented by Daly et al. (1981)). The flux levels are closely similar to those observed during the previous hour when the satellite was in the exterior cusp (see Fig. 1). Figure 4 shows the angular distribution of energetic protons observed during the FTE-related burst at 22:55-22:57 UT (see Fig. 2). The angular distribution shows the proton flux in the azimuth angle-polar angle plane, the angles depicting the flow direction of the particles. Azimuth angle $0^{\circ}$ points to the Sun and increases towards dusk while the polar angle of $90^{\circ}$ points to the direction of GSE-Z axis. The distribution shows that the maximum fluxes are observed around the direction $250^{\circ}$ of azimuth angle and at about $50^{\circ}$ of polar angle, while very low fluxes are observed in the opposite direction. Due to the corrupted central detector heads of the RAPID IIMS instrument we lack data from the four polar bins around the spin plane (polar angles from $-30^{\circ}$ to $30^{\circ}$ ). Despite of this permanent data gap the underlying pattern in the angular distribution is clear. Taking into account the fact that the average magnetic field observed by $\mathrm{C} 4$ is $\boldsymbol{B}_{G S E}=(9.7,31,-25) \mathrm{nT}$ during 22:55:21-22:57:29 UT, i.e., about $72^{\circ}$ from the $\mathrm{X}$ axis towards the evening sector and about $39^{\circ}$ downwards from the XY-GSE plane, the angular distribution depicts a broad ion beam flowing roughly antiparallel to the local magnetic field. Figure 4 also shows overlaid pitch angle contours, which have been calculated in GSE coordinate system using the above mentioned average $\mathrm{C} 4$ magnetic field. The pitch angle contours show even more clearly that the distribution depicts an antiparallel ion beam. Similar distributions were observed during nearly all FTE signatures. Note that during this time the $\mathrm{C} 4$ was in the magnetosheath as indicated by the direction of the magnetic field. Taking into account the direction of the local magnetic field the flow direction of the protons implies that they are coming from the expected reconnection site (magnetopause close to the dusk edge of the cusp funnel) along the field lines and going towards the exterior cusp. 

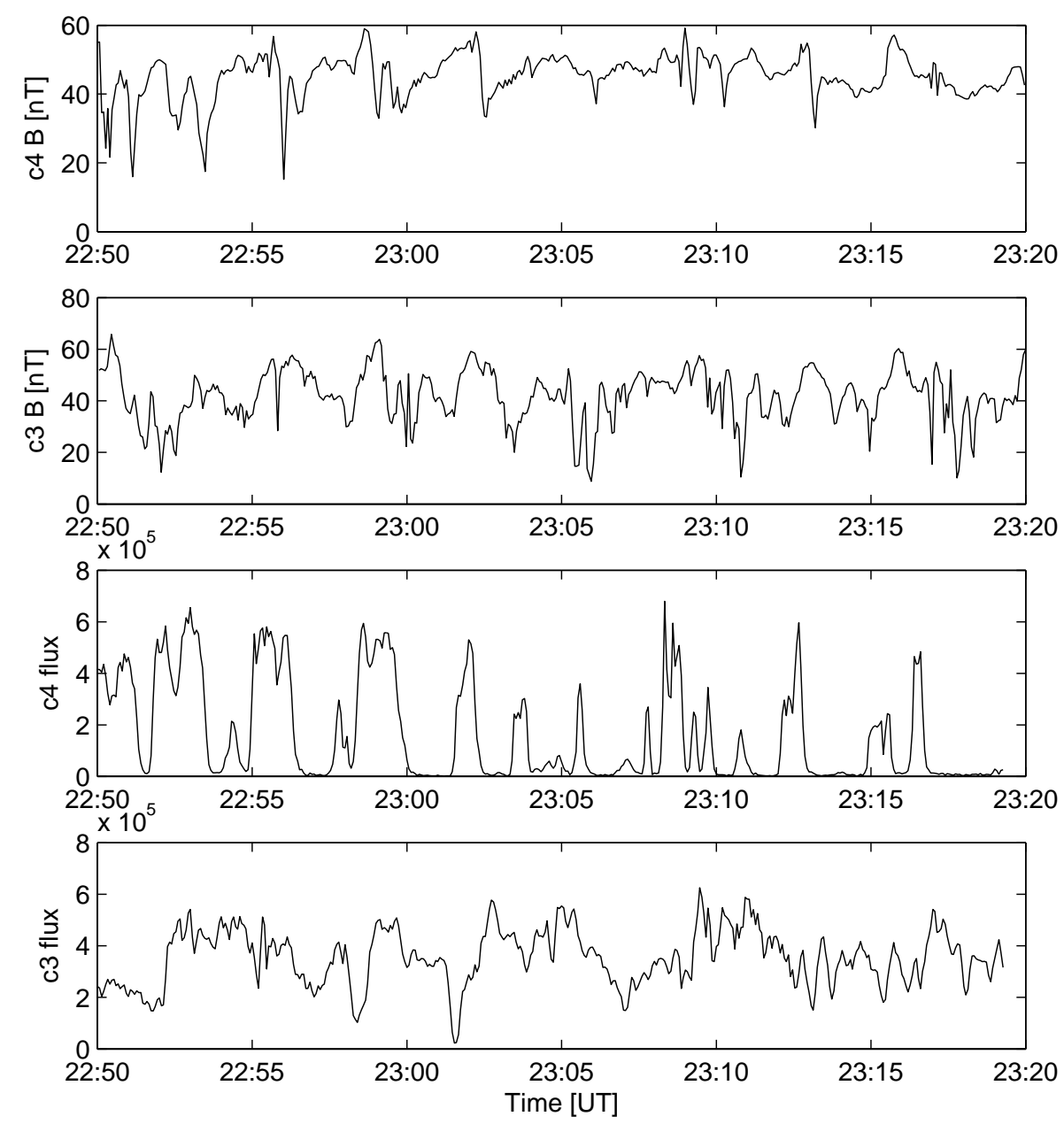

Fig. 5. The magnetic field intensities and the total proton fluxes (in units of $1 / \mathrm{cm}^{2} \mathrm{srs}$ ) measured by the Cluster 4 (first and third panel) and Cluster 3 (second and fourth panel) satellites.
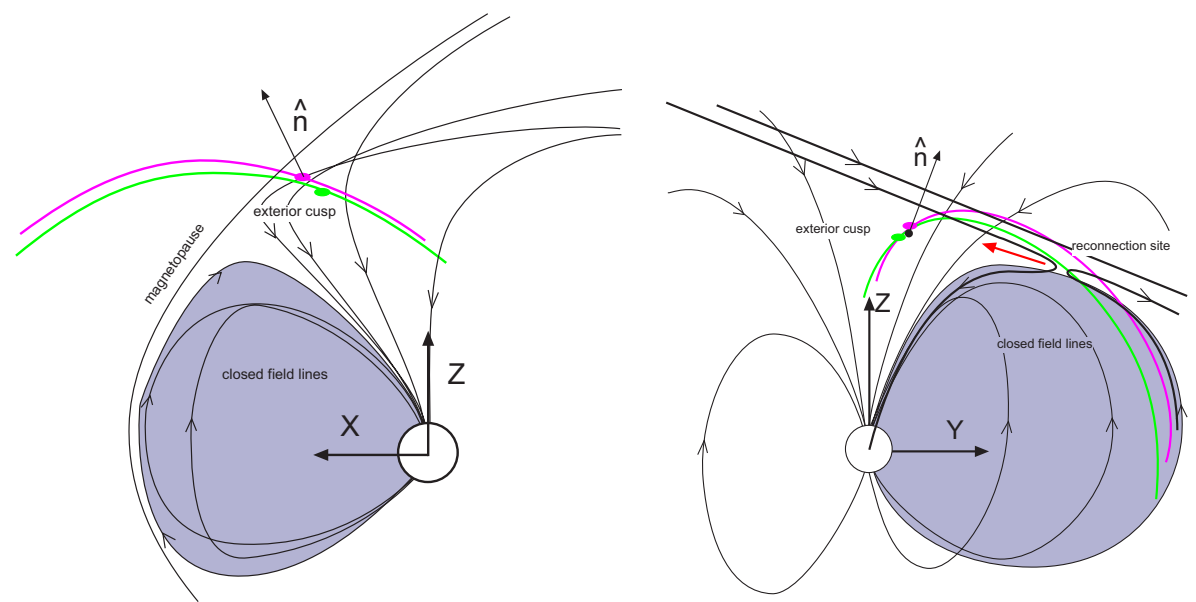

Fig. 6. Schematic diagram of Cluster 3 and 4 satellites and their orbits in the dayside magnetospheric environment. The diagram also shows the estimated boundary normal vector, IMF field lines and the antiparallel reconnection site. The red arrow shows the streaming direction of energetic particles released from the closed field lines in the reconnection. 


\section{Discussion}

As discussed above, Cluster 4 observations just outside the exterior cusp showed bursts of highly enhanced field-aligned fluxes of energetic particles closely associated with FTE signatures in the magnetic field. The observed antiparallel pitchangle distribution indicates that the particles flow from the reconnection site towards the exterior cusp along the field lines. A schematic diagram of the Cluster 3 and 4 orbits and magnetospheric regions is shown in Fig. 6. The $\mathrm{C} 3$ satellite was still deeper in the exterior cusp, close to the boundary between magnetosheath and exterior cusp all the time in 22:50 23:20 UT when C4 observes the FTEs outside the cusp. This is verified, e.g., by the clock angle measurements (Fig. 3) discussed above and lower C3 magnetic field intensity (see Fig. 5) which also depicts dramatic dropouts, typical for the exterior cusp, during all this interval. Figure 5 also shows the energy integrated proton fluxes from the two satellites during this time interval. The third panel shows the FTE related proton flux enhancements observed by $\mathrm{C} 4$ and the bottom panel shows the proton fluxes related to the exterior cusp observed by $\mathrm{C} 3$. One can see that, on top of a rather high background flux level, C3 sees enhancements with some time delay compared to the enhancements observed by $\mathrm{C} 4$.

This is even more clearly demonstrated in Fig. 7 where the correlation coefficient between $\mathrm{C} 3$ and $\mathrm{C} 4$ proton fluxes is shown as a function of the lag between the signals. The first two correlation maxima are found at $40 \mathrm{~s}$ and $3.5 \mathrm{~min}$ time lags. The $40 \mathrm{~s}$ lag maximum corresponds to the average delay between the bursts at $\mathrm{C} 4$ and $\mathrm{C} 3$. The $3.5 \mathrm{~min}$ lag maximum gives the average burst repetition period. The enhancements observed by $\mathrm{C} 3$ are not as well structured as the ones seen by $\mathrm{C} 4$, but this is understandable because $\mathrm{C} 3$ is still observing energetic particles associated to the very dynamic exterior cusp with a large average flux levels. The correlation between the fluxes is not perfect or even expected since the particles come to the cusp from many reconnected flux tubes that each give a different contribution to the total flux in the cusp. The significance of the correlation coefficient is above 99\% at the cross correlation maxima (the corresponding Pvalue, i.e., the probability of zero correlation is below $1 \%$ ). Thus, even though the correlation between $\mathrm{C} 3$ and $\mathrm{C} 4$ fluxes is small the significance is rather high. The comparison between the two satellites (in addition to the $\mathrm{C} 4$ observations) thus strongly suggests that at least part of the energetic particles flowing from the reconnection site can reach the exterior cusp.

Asikainen and Mursula (2005) suggested that the energetic protons in the exterior cusp come from the closed field lines of the high-latitude dayside plasma sheet (HLPS), i.e., the region of closed field lines just below the cusp region. As shown there, the energetic particle fluxes are always nearly an order of magnitude larger in HLPS than in the adjacent exterior cusp. The low-energy $(30-100 \mathrm{keV})$ protons that dominate the total proton fluxes of the RAPID instrument were

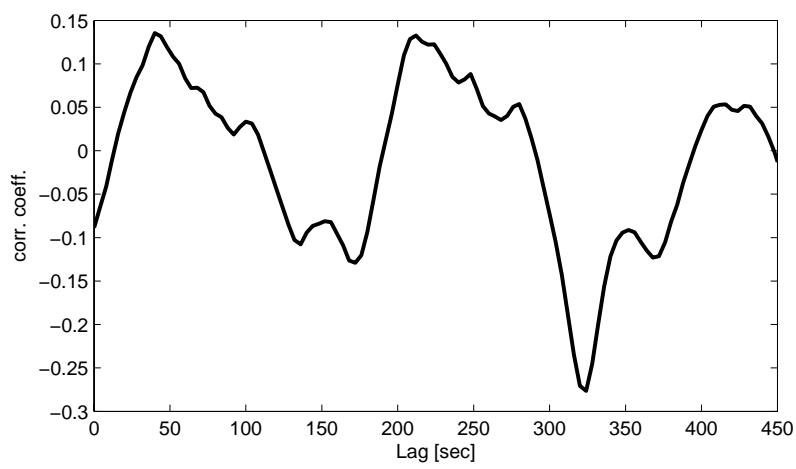

Fig. 7. The correlation coefficient between $\mathrm{C} 3$ and $\mathrm{C} 4$ proton fluxes as a function of the lag between the signals. The time interval is the same as in Fig. 5, i.e., 22:50 to 23:30 UT. Positive lags correspond to shifting of $\mathrm{C} 4$ signal forward in time.

found to depend on the IMF direction, indicating that reconnection might control the access of these particles into the exterior cusp. The observations presented here support this idea showing that energetic particles flow away from the reconnection site and that a part of them arrives in the exterior cusp. In order to rule out the possibility that the particles are energized by reconnection to the observed energies we have estimated the energy per particle obtained in the reconnection process. The observed local plasma density at the satellite is about $10 \mathrm{~cm}^{-3}$ and the local magnetic field intensity about $40 \mathrm{nT}$ in the magnetosheath whence the maximum bulk energy gain per particle in reconnection is about $400 \mathrm{eV}$ assuming that all magnetic field energy is converted to thermal energy of the plasma. This is of course just an order of magnitude estimate since we do not know the exact plasma density and magnetic field in the reconnection region. However, on the basis of these values it is clear that the particles cannot be energized to tens or hundreds of $\mathrm{keVs}$ in the reconnection process by bulk acceleration. Instead, the reconnection process releases part of the pre-existing population of energetic particles from the adjacent closed field lines of the HLPS region.

\section{Conclusions}

We have presented here a case study of flux transfer events observed at the edge of the exterior cusp region by Cluster satellites. Bursts of high fluxes of energetic particles were observed by Cluster 4 in close conjuction with the magnetic field FTE signatures. The pitch angle distribution of the energetic particles showed that the enhancements consist of particles flowing antiparallel to the field lines away from the expected reconnection site towards the exterior cusp. At the same time Cluster 3 observed enhancements of energetic particles inside the exterior cusp with a delay of about $40 \mathrm{~s}$ to the Cluster 4 enhancements. The estimated maximum energy 
gain per particle by reconnection remains below $1 \mathrm{keV}$, thus clearly below the tens to hundreds of keV energy range observed by the RAPID instrument. These observations support the earlier statistical results of the magnetospheric origin of energetic particles in the exterior cusp. Reconnection near the exterior cusp partly releases the protons in the closed field lines of the adjacent HLPS region into the exterior cusp.

Acknowledgements. This work was partly funded by the Academy of Finland. We wish to thank P. Daly for useful discussions and for providing the calibrated RAPID data. We also thank the PIs of the Cluster FGM and CIS instruments, E. Lucek and H. Rème for providing the data.

Topical Editor I. A. Daglis thanks P. Daly and G. Paschmann for their help in evaluating this paper.

\section{References}

Asikainen, T. and Mursula, K.: Energetic particle fluxes in the exterior cusp and the high-latitude dayside magnetosphere: Statistical results from the Cluster/RAPID instrument, Ann. Geophys., 23, 2217-2230, 2005.

Balogh, A., Dunlop, M. W., Cowley, S. W. H., Southwood, D. J., et al.: The Cluster Magnetic Field Investigation, Space Sci. Rev., 79, 65-91, 1997.
Chen, J., Fritz, T. A., Sheldon, R. B., et al.: Cusp energetic particle events: Implications for a major acceleration region of the magnetosphere, J. Geophys. Res., 103, 69-78, 1998.

Daly, P., Williams, D., Russell, C., and Keppler, E.: Particle Signature of Magnetic Flux Transfer Events at the Magnetopause, J. Geophys. Res., 86, 1628-1632, 1981.

Fritz, T. A.: The Cusp as a source of magnetospheric energetic particles, currents, and electric fields: A New Paradigm, Space Sci. Rev., 95, 469-488, 2001.

Mursula, K., Kerttula, R., Asikainen, T., Friedel, R., Vaivads, A., Soeraas, F., Grande, M., Daly, P. W., Fritz, T. A., Fennell, J. F., and Balogh, A.: CLUSTER/RAPID energetic electron observations at the dayside magnetospheric boundary, Adv. Space. Res., 36, 1904-1908, 2005.

Rème, H., Bosqued, J. M., Sauvaud, J. A., et al.: The Cluster Ion Spectrometry (CIS) Experiment, Space Sci. Rev., 79, 303-350, 1997.

Roederer, J. G.: Dynamics of geomagnetically trapped radiation, Springer-Verlag Berlin Heidelberg New York, 66, 1970.

Russell, C. T. and Elphic, R. C.: Initial ISEE magnetometer results: magnetopause observations, Space Sci. Rev., 22, 681-715, 1978.

Sheldon, R. B., Spence, H. E., Sullivan, J. D., Fritz, T. A., and Chen, J.: The discovery of trapped energetic electrons in the outer cusp, Geophys. Res. Lett., 25, 1825-1828, 1998.

Wilken, B., Axford, W. I., Daglis, I., Daly, P., et al.: RAPID: The Imaging Energetic Particle Spectrometer on Cluster, Space Sci. Rev., 79, 399-473, 1997. 